\title{
Pulsed thermography in the investigation of PCBs for defect detection $\&$ analysis
}

\author{
by N.P. Avdelidis, P.I. Nicholson and P. Wallace \\ NDT Group, TWI Technology Centre in South Wales, ECM², Port Talbot, SA13 2EZ, \\ United Kingdom
}

\begin{abstract}
Pulsed thermography (PT) was used for the investigation of printed circuit boards (PCBs) for defect detection and analysis, with the intention of developing a comprehensive in-line quality control system. The overall aim was to develop a new and novel thermal Non Destructive Testing \& Evaluation (NDT \& E) approach for defect detection, providing real time images of modern IC assemblies. Different PCBs containing defects were examined by using PT. Quantification analysis was also implemented; temperature - time plots, as well as thermal contrast curves. Results indicate that PT is a prompt thermal NDT \& E approach that could be used (with some limitations taken into consideration) in the assessment of PCBs.
\end{abstract}

\section{Introduction}

It is already standard practice in the $\mathrm{PCB}$ industry to use IR imaging technology to locate failures in populated PCBs [1]. This typically involves temporarily applying power to the PCB to identify short circuits, and using passive thermography to image the resulting heat dissipation. However, this process has the possibility to be destructive, if the degree of defect is serious.

European environmental legislation, to be implemented in 2006 banning the use of lead, mercury, cadmium and hexlent chromium, PPB and PBDE (two types of brominated flame retardants) has the potential to increase further the likelihood of such defects and introduce new types of defects. The melting point of all lead free solders is much higher than the previously used tin/lead alloy. This extra temperature can result in extra stressing of the components and PCB. Damage can include PCB warping, causing damage to components, component misalignment, damage to heat sensitive components, and delamination of multi-layer PCBs. The passive thermography approach is not suited to detecting these types of defects and in particular PCB delamination, where a separation between plies within the base material or between the base material and the conductive or both is formed.

This paper presents the application of a pulsed delivery system for active thermography imaging of PCBs.

In thermal NDT \& E, the active approach of infrared thermography where an excitation source, such as optical flash lamps, heat lamps, hot or cold air guns, etc., is employed with the intention of inducing thermal contrasts, has several applications [2-5]. The temperature differences during the transient phase appear on the material surface and so detection of subsurface defects is possible (areas of different temperatures when compared to the sound part(s) due to the different thermal diffusivity). Since the heating or cooling features of the stimulus source are 
identifiable (in time and amplitude) by considering the time factor quantitative assessment is also feasible. However, when a material is heated, the thermal waves penetrate the material's surface. These waves are generally of various amplitudes and frequencies and are launched into the specimen, in a transient mode.

The most common modes of active thermography are pulsed thermography, long pulsed heating or step heating transient thermography, optical lock-in thermography, and ultrasound lock-in thermography.

PT is one of the widely used approaches for active infrared thermographic NDT \& E. In this technique, also known as transient thermography, the surface under investigation is pulse heated (time period of heating varying from ms for sort pulse or flash thermography to a few s for long pulse thermography) by one or more powerful optical lamps and the resulting thermal transient at the surface is monitored using an infrared camera. Nonetheless, the duration of the heating pulse depends on the thermal and physical properties of the materials, as well as its thickness. So, the heat flow into the sample is altered in the presence of a subsurface defect or feature, creating temperature contrast at the surface and as a result can be detected recorded by an infrared system [6].

\section{Experimental procedures and techniques}

Two different integrated pulsed thermographic systems (Thermoscope and Echotherm) were used. In both instances, medium wave infrared cameras were used; a Merlin 3-5 $\mu \mathrm{m}$ with the Thermoscope system and a Phoenix 3-5 $\mu \mathrm{m}$ with the Echotherm. Both systems are portable state-of-the-art NDT \& E systems with integrated flash heating ability.

Both systems allow (through the software) the processing of the thermal images and the production of temperature - time plots, line profiles (on both the $X$ and $Y$ axes of an image), 3-D plots (in order to identify subtle image features that may not be apparent in the 2-D image) and reconstructed $1^{\text {st }}$ and $2^{\text {nd }}$ time derivative images. A total of eight samples - circuit boards were manufactured and examined.

Table 1. Description of investigated samples - circuit boards

\begin{tabular}{|c|c|}
\hline Sample & Description of Sample \\
\hline S1 & $\begin{array}{c}\text { Circuit Board with heat plane delamination on the } \\
\text { board }\end{array}$ \\
\hline $\begin{array}{c}\text { S2, S4, } \\
\text { CJ8 \& CJ9 }\end{array}$ & $\begin{array}{c}\text { Circuit Board with various types of soldering defects } \\
\text { (i.e. capacitor, chip resistor, etc) }\end{array}$ \\
\hline S9, S10 & $\begin{array}{l}\text { Circuit Board with visible heat plane delamination on } \\
\text { the board }\end{array}$ \\
\hline
\end{tabular}

Representative thermal images of the investigated samples were obtained during the transient phase of the thermographic inspections and are presented. In some instances, plots of the contrast versus the transient time of the identified defects were performed; the difference in intensity ( $\Delta$ Intensity) between the detected defect and the sound region of the sample against the transient time during the cooling down process. 


\section{Results and discussion}

After investigating sample $\mathrm{S} 1$, it was possible to image a delaminated area on the board. A thermal contrast ( $\Delta$ Intensity) curve between the delaminated area and a sound area within the board is also presented (i.e. this is against the transient time during the cooling down process).

Furthermore, after investigating sample S4, it was possible to image soldering defects (i.e. capacitor, chip resistor). Nonetheless the problem of reflectivity with thermography (see top left part of image shown in figure 5) is an issue to be considered. Similar results were attained after the investigation of samples S2, CJ8 and CJ9.

Finally, in the case of samples S9, S10 and S11, things were very straightforward. PT very easily detected the delaminations on the board.

Sample SAM1 - Delaminated Area

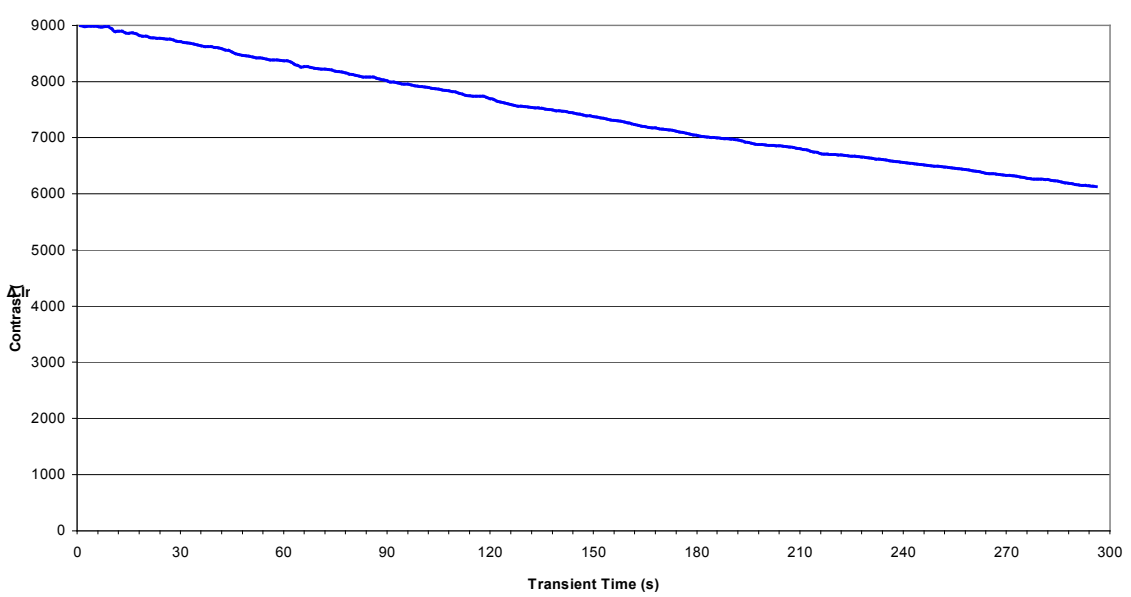

Fig. 1. Contrast curve between delaminated area and sound area of sample S1

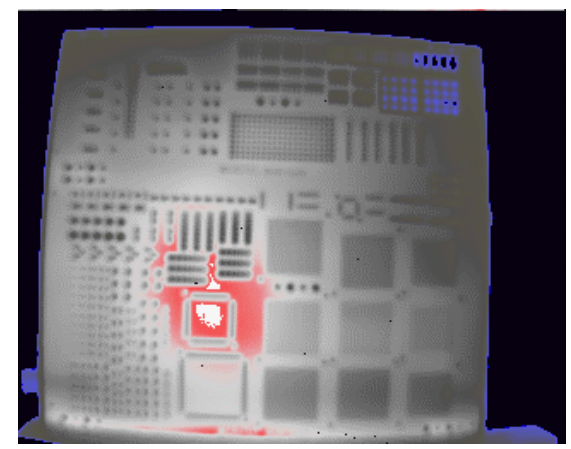

Fig. 2. Sample $\mathrm{S} 1$ at a frame rate of $7.5 \mathrm{~Hz}$. Image acquired at 4.275 seconds indicating delaminated area on the board 


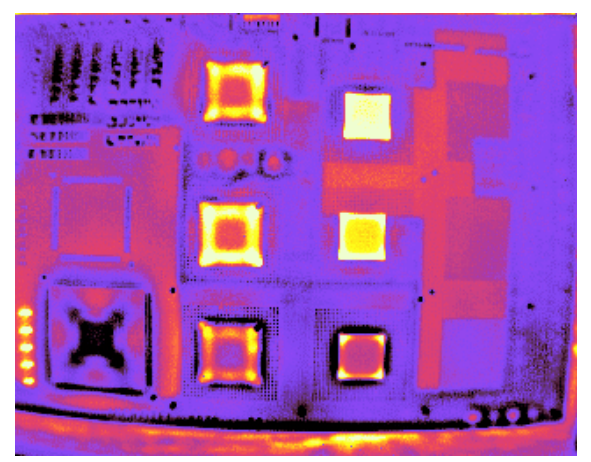

Fig. 3. Sample $\mathrm{S} 4$ at a frame rate of $15 \mathrm{~Hz} .2^{\text {nd }}$ time derivative image acquired at 7.415 seconds revealing soldering defects

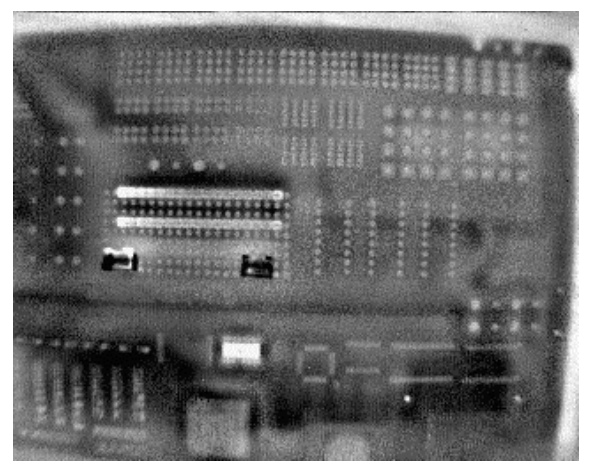

Fig. 4. Sample $\mathrm{S} 4$ at a frame rate of $15 \mathrm{~Hz} .2^{\text {nd }}$ time derivative image acquired at 17.435 seconds showing a soldering defect

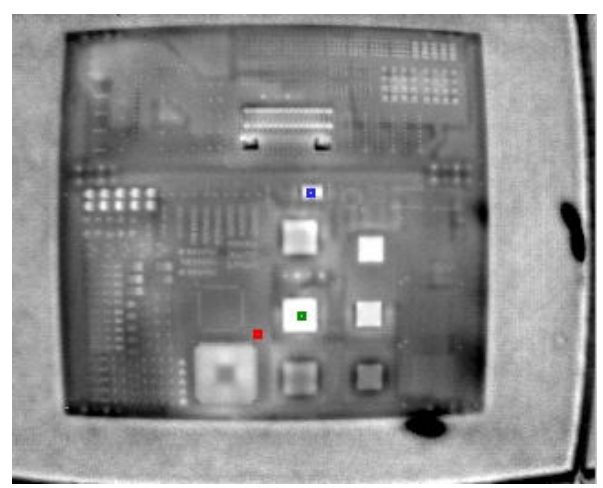

Fig. 5. Sample $\mathrm{S} 4$ at a frame rate of $15 \mathrm{~Hz} .2^{\text {nd }}$ time derivative image acquired at 14.482 seconds 


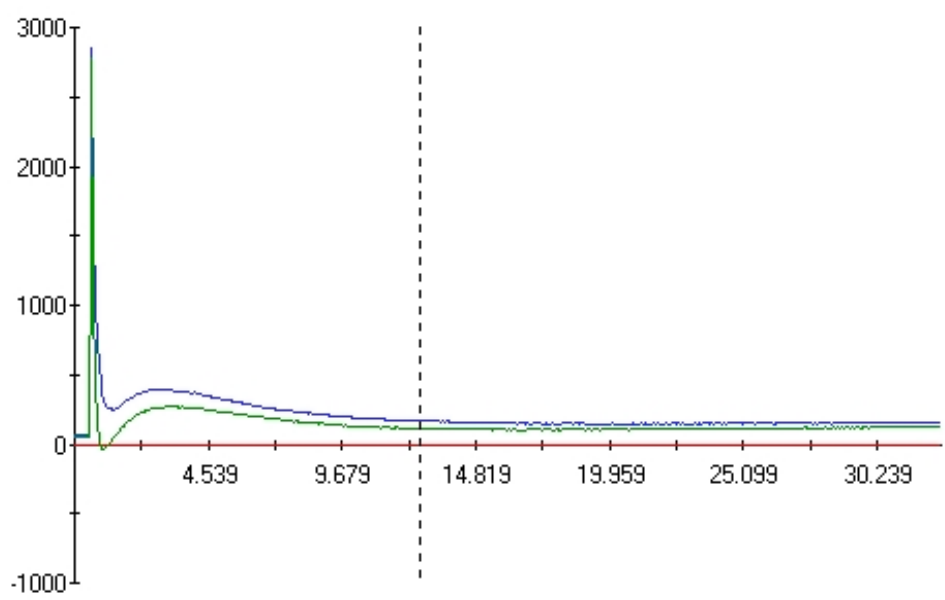

Fig. 6. Contrast curves from points selected in sample S4 from figure 5

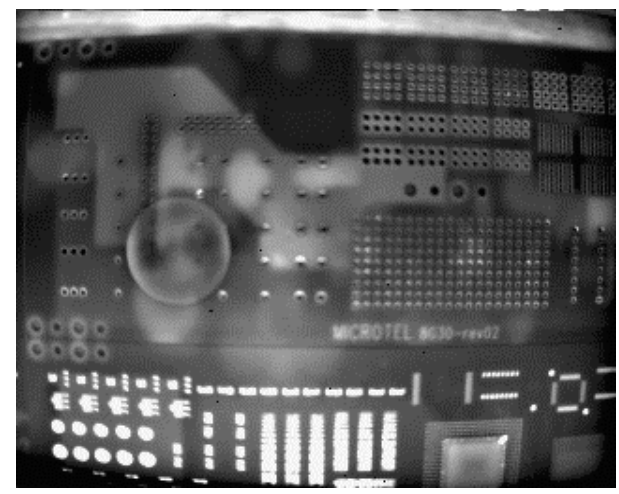

Fig. 7. Sample $\mathrm{S} 9$ at a frame rate of $60 \mathrm{~Hz}$. Image acquired at 1.603 seconds revealing delaminations on the board

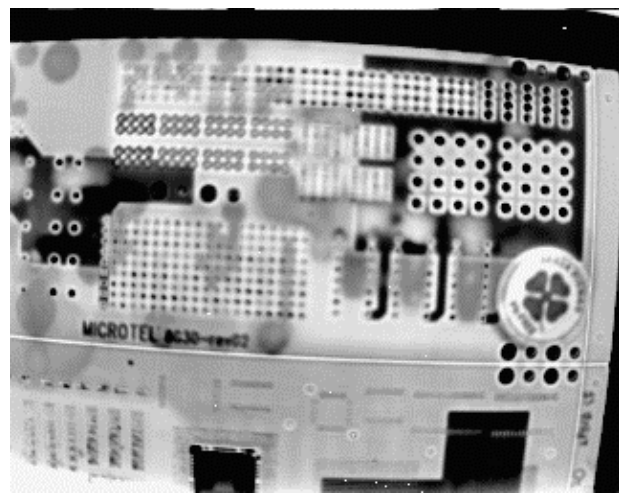

Fig. 8. Sample $S 9$ at a frame rate of $60 \mathrm{~Hz}$. 1st time derivative image acquired at 0.551 seconds revealing delaminations on the board 


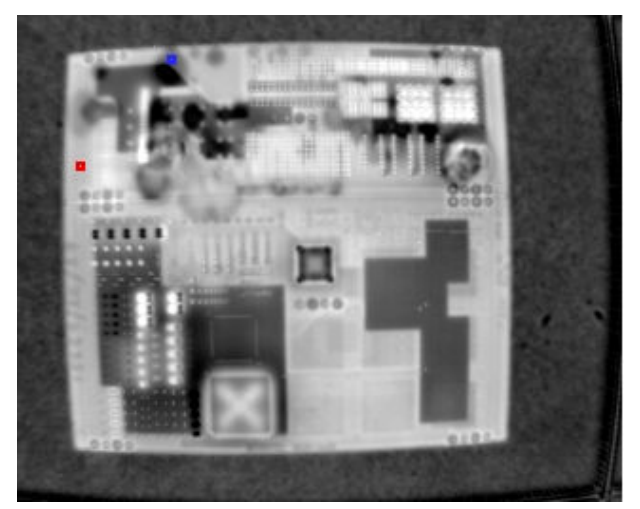

Fig. 9. Sample $\mathrm{S} 9$ at a frame rate of $60 \mathrm{~Hz} .2^{\text {nd }}$ time derivative image acquired at 2.720 seconds revealing delaminations on the board

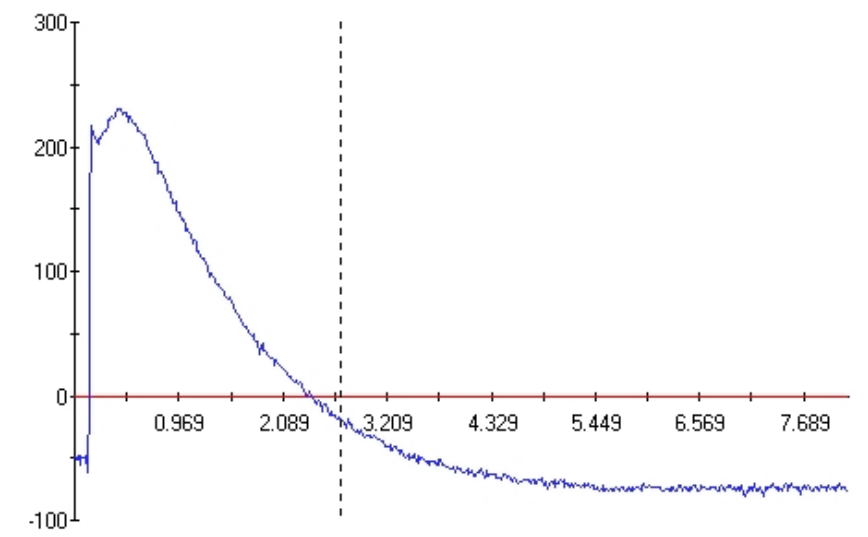

Fig. 10. Contrast curves from points selected in sample S9 from figure 9

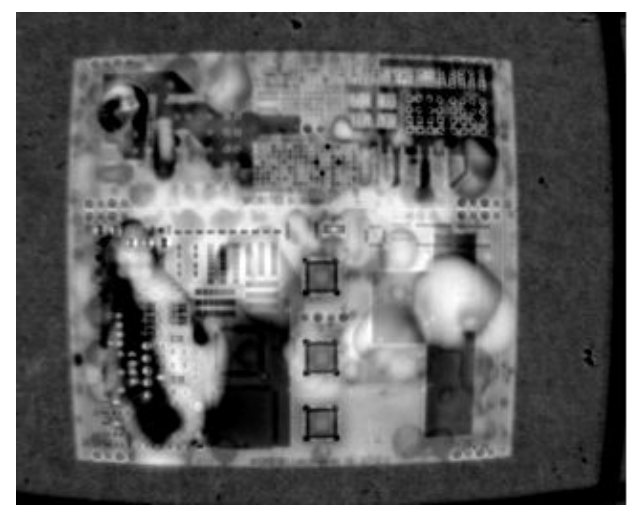

Fig. 11. Sample $\mathrm{S} 10$ at a frame rate of $15 \mathrm{~Hz} .1^{\text {st }}$ time derivative image acquired at 1.135 seconds revealing delaminations on the board 


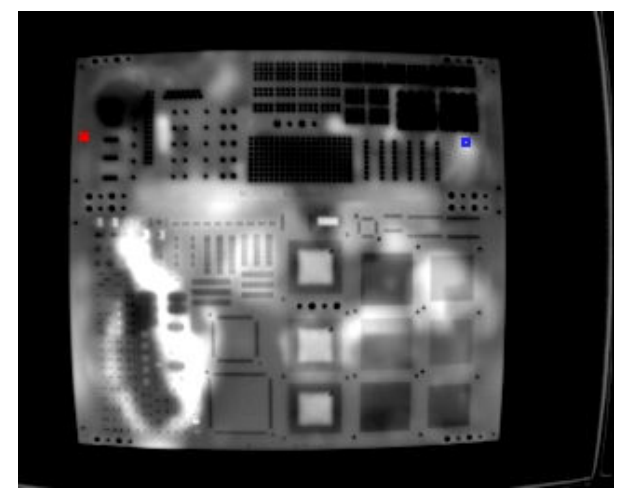

Fig. 12. Sample $\mathrm{S} 10$ at a frame rate of $60 \mathrm{~Hz}$. Original image acquired at 4.555 seconds revealing delaminations on the board

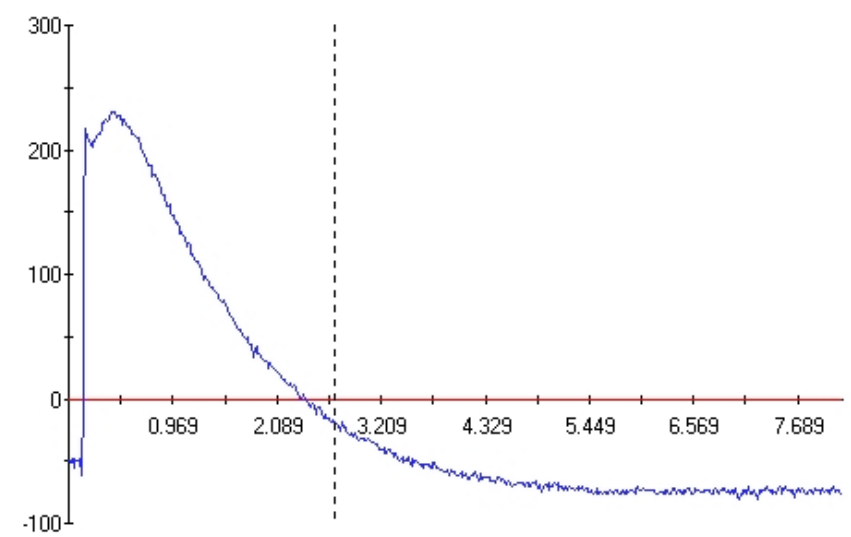

Fig. 13. Contrast curves from points selected in sample S10 from figure 12

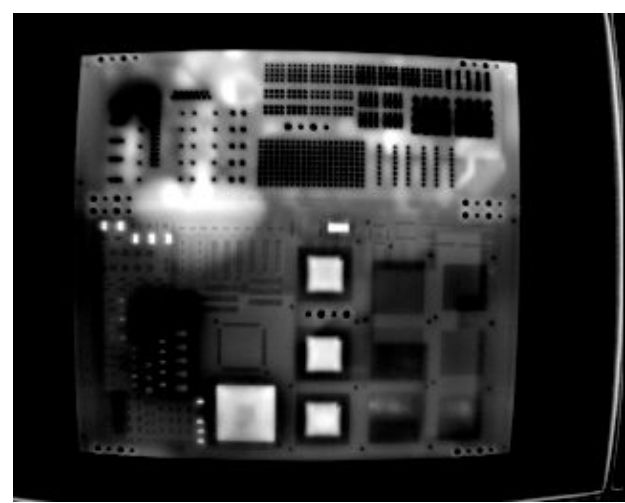

Fig. 14. Sample $\mathrm{S} 11$ at a frame rate of $60 \mathrm{~Hz}$. Original image acquired at 2.870 seconds showing delaminations on the board 
Since the thermal conductivity of all these tested samples is relatively low, the samples were tested using a reasonably low maximum frame rate. In particular, a frame rate of either $7.5 \mathrm{~Hz}$ or $15 \mathrm{~Hz}$ was used. Furthermore, in the case of samples S9, S10 and S11, since the damage was mostly on the surface (delaminations) a higher frame rate $(60 \mathrm{~Hz})$ in addition to the already used frame of $15 \mathrm{~Hz}$ was employed.

\section{Summary \& Conclusions}

Transient - pulsed thermography has emerged in recent years as a means of NDT \& $E$ technique. Its advantages are that it is a rapid large area non-contact imaging technique that produces images of subsurface features (i.e. defects) that are relatively straightforward to interpret. In a few words, the area to be inspected is heated by high intensity optical lamps and an infrared camera monitors the subsequent thermal transients across the area.

Trials of PT took place on different PCBs containing various defects. In some instances, the technique provided prompt results. Nonetheless, in some instances the problem of reflectivity within transient thermography investigation needs to be considered. Furthermore, the different thermal properties of the material under investigation (different behaviour between high thermal conductive materials such as metals and lower thermal conductive materials such as composites), need to be considered as far as the frame rate of image acquisition is to take place (i.e. in a fully automated NDT \& E investigation system). Nonetheless, the results of this work indicate that PT can be used in the detection of defects (i.e. delaminations and/or soldering defects) when investigating circuit boards.

An integrated flash heating system (like either of the two used in this work) could be used for testing such circuit boards. For this reason, there is really no need to make extra considerations concerning the orientation of thermal excitation source(s) and the camera sensitivity issue. The methodology for selecting the appropriate frame rate on an integrated thermographic system (i.e. pulsed - flash thermographic equipment) it is entirely dependant on the experience and knowledge of the thermography operator.

After subjecting a PCB to thermal stress, it would be possible to examine the thermal profile and use that profile for comparing trends during the manufacturing process. For example over time, a batch of components may have slightly increasing differing functionality with respect to temperature, or a batch of multi-layer PCBs may become delaminated due to an accumulative small change in the manufacturing process. Using active thermography, these changes could be detected and used to predict component/PCB failure.

\section{ACKNOWLEDGEMENTS}

The results from this work are part of the CRAFT programme: Development of comprehensive in-line quality control system for printed circuit board assemblies, Acronym MICROSCAN, COOP-CT-2003-508613. MICROSCAN is a collaboration between the following organisations: TWI Ltd, X-TEK Systems Ltd, Lot Oriel GmbH, Machine Vision Products Inc, Microtel Technologie Elettroniche SpA, Beta Electronics Ltd, Ultrasonic Sciences Ltd, Goodrich Control Systems Ltd, FraunhoferGesellschaft zur Foerderung der Angewandten Forschung E.V. and Kaunus 
University of Technology. The project is co-ordinated and managed by TWI Ltd and is partly funded by the EC.

Additional thanks are attributed to Microtel Technologie Elettroniche SpA for manufacturing the PCBs and preparing the defects. Finally, acknowledgements are attributed to Prof. Darryl Almond, Materials Research Centre, University of Bath, for using the Thermoscope equipment.

\section{REFERENCES}

[1] C. Alicandro, and D. Little. Thermography in the microelectronics industry, Evaluation Engineering, on-line articles (1999).

[2] N.P. Avdelidis, D.P. Almond, A. Dobbinson, B.C. Hawtin, C. Ibarra-Castanedo, and X. Maldague. Invited Review Paper: Aircraft composites assessment by means of transient thermal NDT, Progress in Aerospace Sciences, 40 (2004)143-162.

[3] N.P. Avdelidis, E.T. Delegou, D.P. Almond, and A. Moropoulou. Surface roughness evaluation of marble by $3-D$ laser profilometry and pulsed thermography, NDT \& E International, 37 (2004) 571-575.

[4] N.P. Avdelidis, A. Moropoulou, and D. Stavrakas. Detection and quantification of discontinuities in building materials using transient thermal NDT techniques: modelling and experimental work, Materials Evaluation, 64 (2006) 489-491.

[5] J.M. Milne, and W.N. Reynolds. The non-destructive evaluation of composites and other materials by thermal pulse video thermography, Thermosense VII, 520 (1984) 119-122.

[6] N.P. Avdelidis, B.C. Hawtin, and D.P. Almond. Transient thermography in the assessment of defects of aircraft composites, NDT \& E International, 36 (2003) 433-439. 